\title{
Pressure and Temperature Effect on Hydrogen Sorption of Carbon Material Containing Nanotubes
}

\author{
GULIDOVA Lyudmila V. ${ }^{a}$, DUBROVA Natalya A. ${ }^{b}$, LIDER Andrey M. $^{\mathrm{c}}$
}

National Research Tomsk Polytechnic University, 30 Lenina Avenue, Tomsk, 634050, Russia

a Igulidova@tpu.ru, ${ }^{\mathrm{b}}$ dubrova@tpu.ru, ${ }^{\mathrm{c}}$ lider@tpu.ru

Keywords: hydrogen storage, carbon nanotubes, sorption, desorption

\begin{abstract}
The paper presents the experimental results of saturation of the carbon material containing nanotubes with hydrogen from the gas. The dependences of hydrogen concentration from pressure (from 0 to 8 atmospheres), at the same temperature for adsorption (-30 degrees Celsius) and different temperatures for desorption were obtained. The temperature has an influence on sorption-desorption process and sorption properties of carbon material. The amount of residual hydrogen depends on saturation parameters.
\end{abstract}

\section{Introduction}

The main question of alternative energetic is searching of effective, environmental safety, available and rather inexpensive energy source. Hydrogen has many necessary properties for satisfaction of all the energy needs of humanity. Firstly, the world reserves of this element are almost inexhaustible. World Ocean contains about $10^{14}$ tons of hydrogen. Secondly, hydrogen is the most energy intensive fuel. Hydrogen specific heat of combustion is $143 \mathrm{~kJ} / \mathrm{g}$. The sense is three times higher than specific heat of combustion of hydrocarbons. Thirdly, the oxidation product of hydrogen is water, which is more environmentally friendly than other kinds of fuel.

Despite all the advantages of hydrogen the problem related with its storage is not completely solved. In the century of nanotechnology the standard methods of hydrogen storage require new materials and improvements. The using of carbon nanotubes as material-accumulator is original way for solving the problems of hydrogen storage [1].

\section{The production of carbon material and purification}

Carbon nanotubes are produced by different methods. The basic methods are arc discharge, laser ablation and chemical deposition. The main disadvantage of the methods is necessity of intensive purification of material. The method of conversion [2] of natural gas was created in Tomsk Polytechnic University (Tomsk) for associated gas utilization. Method makes it possible to synthesize without metal catalytic particles carbon material. Initially this method was developed for producing hydrogen from natural gas, but scientists discovered the strange cylindrical objects in the by-product. The peculiarity of this method is combination of metal catalysts and influence of microwave field on the catalysts and methane. Scientists used the flow reactor of waveguide type. The main difference of this method from other catalytic methods of producing carbon nanomaterial that carbon doesn't cover catalytic particles. Scientists used metals Ni, Fe, Mo, Ti in the capacity of catalysts.

The carbon nanomaterial consists of different modifications of carbon: single- and multi-wall carbon nanotubes, nanofibers, fullerenes, microcrystals of graphite, carbon nanoparticles and amorphous carbon. The content of carbon nanotubes in the final product is near $70 \%$.

From the article the causes of the poor hydrogen sorption by carbon nanotubes are bad sample preparation, for example low-quality purification, functional groups and pore sizes [3]. The process of purification has big influence on hydrogen sorption. Generally, the purification methods can be divided into dry and wet methods. The third standard methods of carbon nanomaterial purification were used. There are settling after ultrasonic dispersion, oxidation in nitric acid and annealing 
material in the vacuum oven. The oxidation process is used for opening the nanotubes, because all nanotubes have hemispherical cap, which prevents to penetration of hydrogen in the nanotubes. The annealing is used for removal of amorphous carbon [4].

\section{Experiments and results}

The samples of carbon material were researched with the help of scanning and transmission electron microscopy. For making the microscopic investigations the sample of the superfine carbon material was mixed with acetone and dispersed in the ultrasonic bath. The resulting suspension was applied on the electron microscope copper mesh with carbon film. Figure 1 (a) shows the micrographs of carbon material which has the large quantity of carbon nanotubes with different diameters. The diameters reach $20-150 \mathrm{~nm}$. The Figure 1 (b) shows a micrograph of the carbon material, which clearly distinguishes are carbon nanotubes having an inner diameter of $25 \mathrm{~nm}$ and wall thickness of about $20 \mathrm{~nm}$.

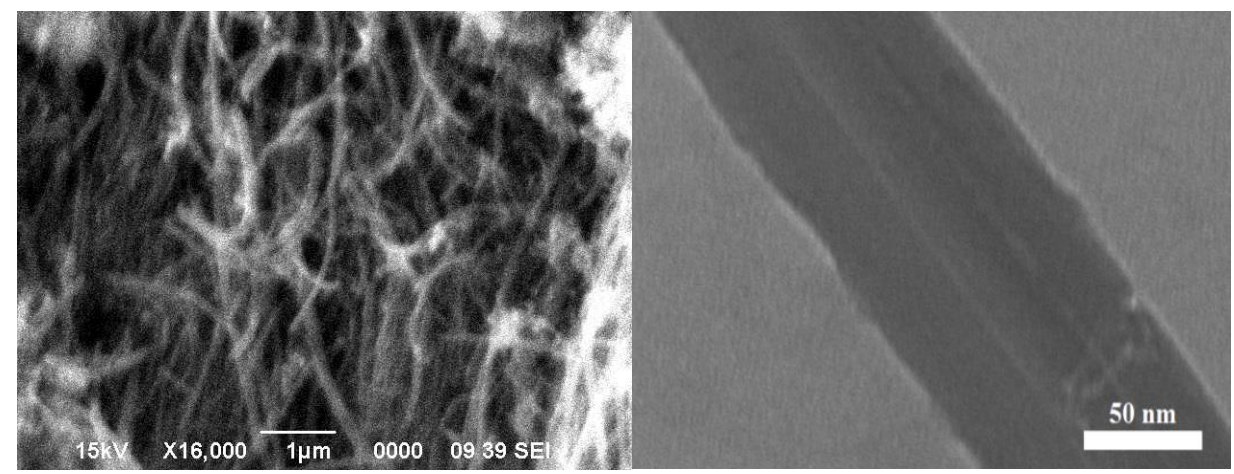

a)

b)

Fig.1. Scanning and transmission micrographs of carbon material:

a) nanotube-clusters b) fragment of single nanotube.

Scientists have some problems with understanding of hydrogen sorption mechanism in carbon nanotubes [5]. The number of wall, diameters and length of carbon nanotubes influence on sorption properties. The metals catalysts or "incrustation" of nanotubes also can improve properties of materials.

In the work the saturation parameters influence on hydrogen capacity of carbon material. A lot of investigation groups published their results of anomalous sorption capacity at very low temperature [6]. The high pressure for increasing of amount absorbed hydrogen at room temperature is needed.

The automatic system Gas Reaction Controller of Advanced Material Corporation was used for measuring the sorption of hydrogen by carbon nanotubes. The detailed description and principle of operation was demonstrated at last work [7].

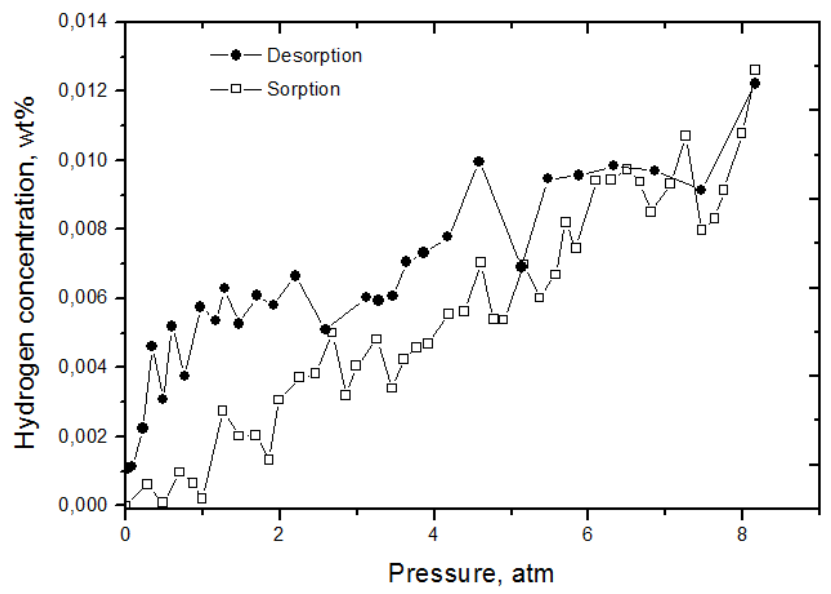

a)

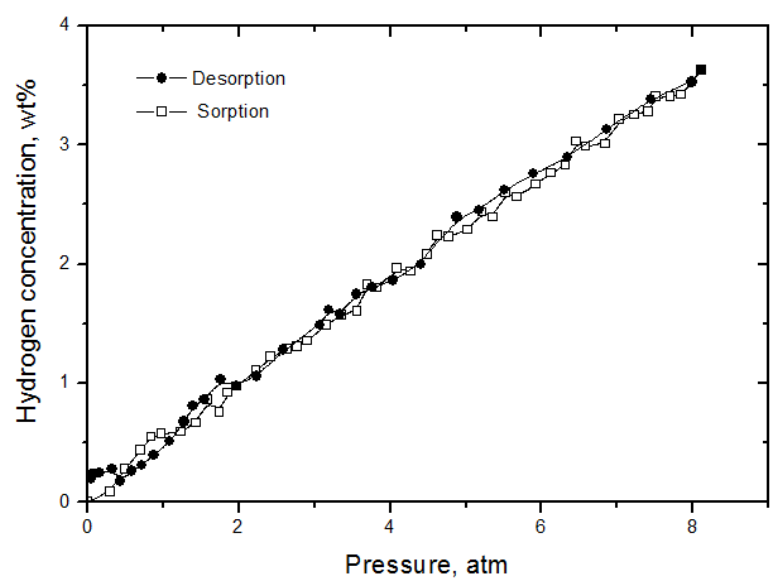

b) 


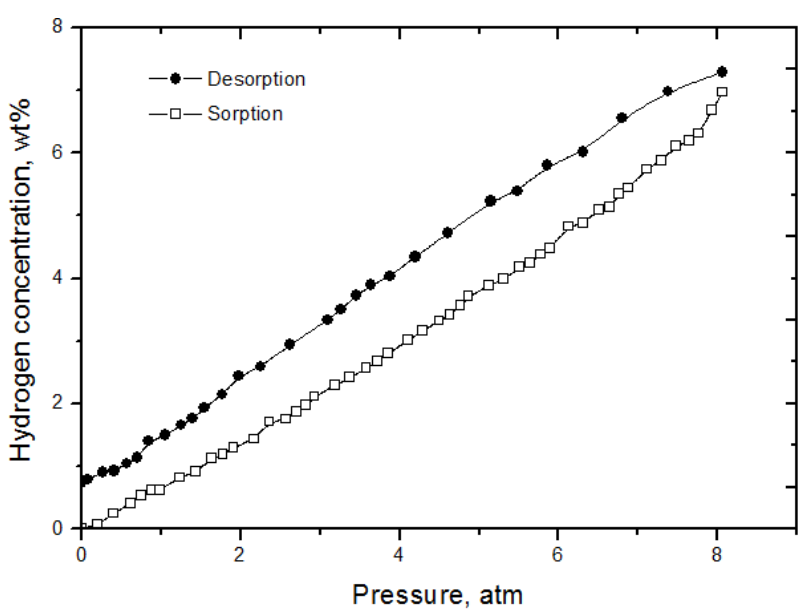

c)

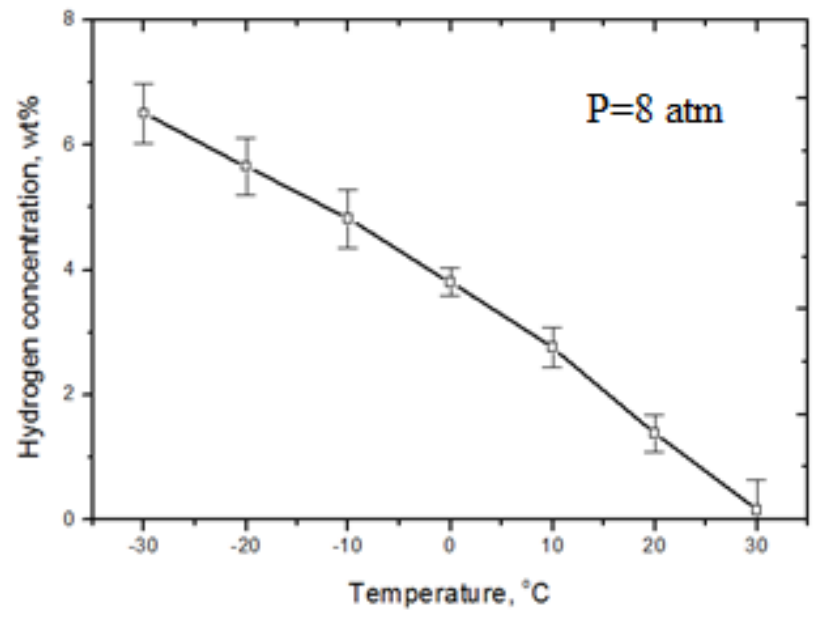

d)

Fig. 2. Concentration-pressure diagram: a) $\left.\left.+30^{\circ} \mathrm{C} \mathrm{b}\right) 0^{\circ} \mathrm{C} \mathrm{c}\right)-30^{\circ} \mathrm{C}$; d) concentration-temperature diagram.

Measurements of absorption and desorption PCI (pressure concentration isotherm) curves and kinetics measurements of carbon nanotubes have been done under the following conditions: the pressure range $0-8 \mathrm{~atm}$. and temperatures $-30{ }^{\circ} \mathrm{C}, 0^{\circ} \mathrm{C}, 30^{\circ} \mathrm{C}$.

The Fig. 2 (a) shows spasmodic changes of hydrogen concentration. This situation does not observe in the next concentration-pressure diagrams (Fig. 2 b,c). The maximum hydrogen sorption capacity of carbon materials is $7 \mathrm{wt} \%$ at high pressure and low temperature. The concentrations of absorbed hydrogen increases with sorption-desorption temperature decrease at maximum pressure (Fig. 2 d). The Fig.2 (a,b,c) also show the residual hydrogen concentration decrease with temperature increasing.

\section{Summary}

Results of experiment show undoubted general sorption parameters influence hydrogen concentration. The using of low temperature and high pressure allows for the achievement of high values of sorption capacity for the effective hydrogen storage.

\section{Acknowledgements}

The authors would like to sincerely thank A.G. Zherlitsyn for provision of carbon material.

\section{References}

[1] Peter J. F. Harris, Carbon Nanotubes and Related Structures. New materials for the Twenty-first Century, Cambridge University Press. (1999) 277.

[2] A.G. Zherlitsyn, V.P. Shiyan, V.S. Kositsyn, L.V. Gulidova, A.M. Lider, P.S. Postnikov, M.E. Trusova, Production of Hydrogen and Carbon Nanomaterials from Natural Gas for Hydrogen Storage Devices, Izvestia vuzov. Fizika. 55 (2012) 230-234.

[3] S.M. Lee, Y.H. Lee., Hydrogen storage in single-walled carbon nanotubes, Appl. Phys. Lett. 20 (2000) 2877-2879.

[4] Xin Xu, Maohong Fan, Brian Francis Towler and other, Hydrogen Adsorption and storage, Elsevier Inc. (2011) 157-245.

[5] W.-C.Xu, K.Takahashi, Y.Matsuo, Y.Hattori, M. Kumagai, S. Ishiyama,K. Kaneko, S. Iijima. Investigation of hydrogen storage capacity of various carbon materials. Int. J. Hydrogen Energy. 32 (2007) 2504-2512. 
[6] Luydmila V. Gulidova, Victor N. Kudiyarov, Andrey M. Lider, The Investigation of Hydrogen Sorption-desorption Process by Carbon Material with Content of Carbon Nanotubes, IFOST. 2 (2012) 58-61.

[7] Viktor N. Kudiiarov, Luydmila V. Gulidova, Natalia S. Pushilina, Andrey M. Lider, Application of automated complex gas reaction controller for hydrogen storage materials investigation, AMR. 740 (2013) 690-693. 